\title{
Fracture Surface Morphology of Cracked Organic Matters in Kerogen-rich Source Rock Observed in Electron Microscope
}

\author{
Mohammad Haque \\ Aramco Americas, Katy, Texas, United States
}

Investigating fracture surface of any materials such as polymers, metals, semiconductors, ceramics, minerals, rocks, or composites, provides valuable information of its failure modes, failure initiation, and cracking behavior. In hydraulic fracturing technology, failure properties of source shale and its individual constituents are studied in tension or compression to better understand the creation of the fracture network and the enhancement of its flow conductivity for economical hydrocarbon production. Source shale is a complex natural composite consisting of clays (i.e., illite, mica, and smectite), non-clay minerals (QFPquartz, feldspar, and pyrites) and organic matter (i.e., kerogen and bitumen) intertwined within the shale matrix. Kerogen is a very stable and insoluble macromolecular geopolymer dispersed in the rock matrix. The elastomer nature of kerogen as well as its viscous-like behavior greatly influences the hydraulic fracture optimization.

In this work, millimeter size single edge notch beam (SENB) were prepared from source shale (Woodford, a source rock from the Texas basin). The fracture surface morphology of the trapped kerogen, after a controlled crack propagated across the bedding plane (Fig. 1a), were imaged by scanning electron microscope (SEM) - both in secondary (SE) and backscattered (BSE) electrons modes. The naturally created fracture surfaces of the cracked kerogen under study had the most preserved fracture morphology. The track of the crack propagation was also remained in its most original state after fracturing the rock matrix. This is because traditional sample preparation techniques for SEM imaging utilizes mechanical polishing or ion milling of the rock surface which alter or damage the exposed kerogen diminishing the surface morphology originated during fracturing process. Unique feature such as obsidian fracture, commonly observed in glassy/brittle minerals like quartz, is reported to be observed in kerogen geopolymer. A combination of BSE and energy dispersive X-ray (EDS) were used to identify the kerogen intertwined in the mineral matrix (Fig. 1b), whereas, SE scanning was used for the topography analysis.

Kerogen, a tough natural geopolymer, undergoes extreme aging at a geological timescale leading to maturation (types I, II, and III) and embrittlement as exposed to temperature and high stress. Fractography of kerogen revealed high yield and high tensile strength failure. Depending upon the shape, size, location and orientation of the kerogen body in the matrix, a kerogen body may or may not be cracked when rock matrix is fractured. Fig. 2 shows a series of examples of glassy fractures for fractured kerogen where crack propagated through the kerogen body across the bedding plane. A mineral inclusion occurred to be trapped within the kerogen body, which became exposed after the crack propagated along its interface, leaving the indentation mark on the cracked kerogen surface (Figs 2a, 2b). In other events, obsidian fracture like microstructures (Figs. 2d, 2e) and a complex multibody multi-planner fracture were observed (Fig. 2e) with three parallel ripple lines, indicating high stress failure. In summary, there is not a single kind of fracture morphology that can generalize the kerogen failure. But a strong high yield high tensile behavior with glassy fracture was common in most kerogen samples studied in this work.

Acknowledgment: The author would like to acknowledge Jordan Kone for SEM imaging and' Younane N. Abousleiman, Rajesh K. Saini, David Jacobi, Katherine Hull, and Yanhui Han for their valuable discussions and input. 

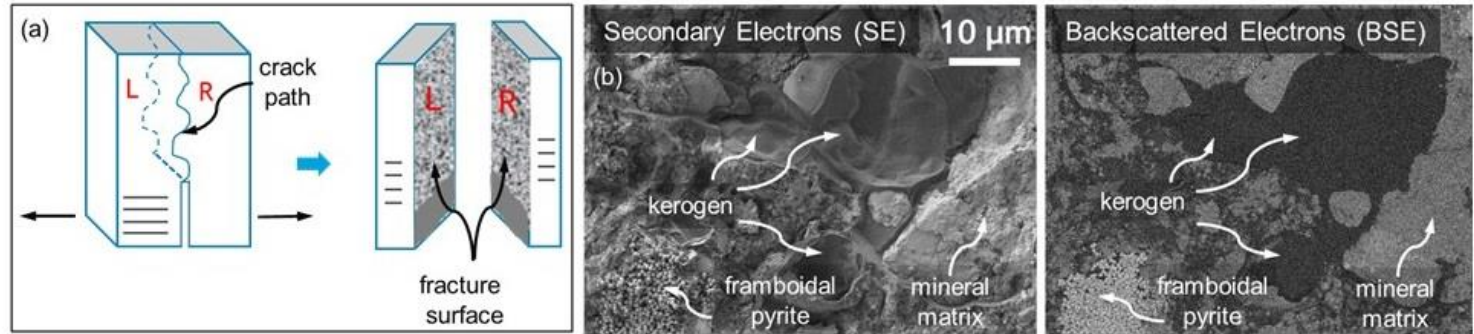

Figure 1. Schematic of prepared samples (a). SE and BSE electron microscopy of a fracture surface showing the presence of kerogen (b).

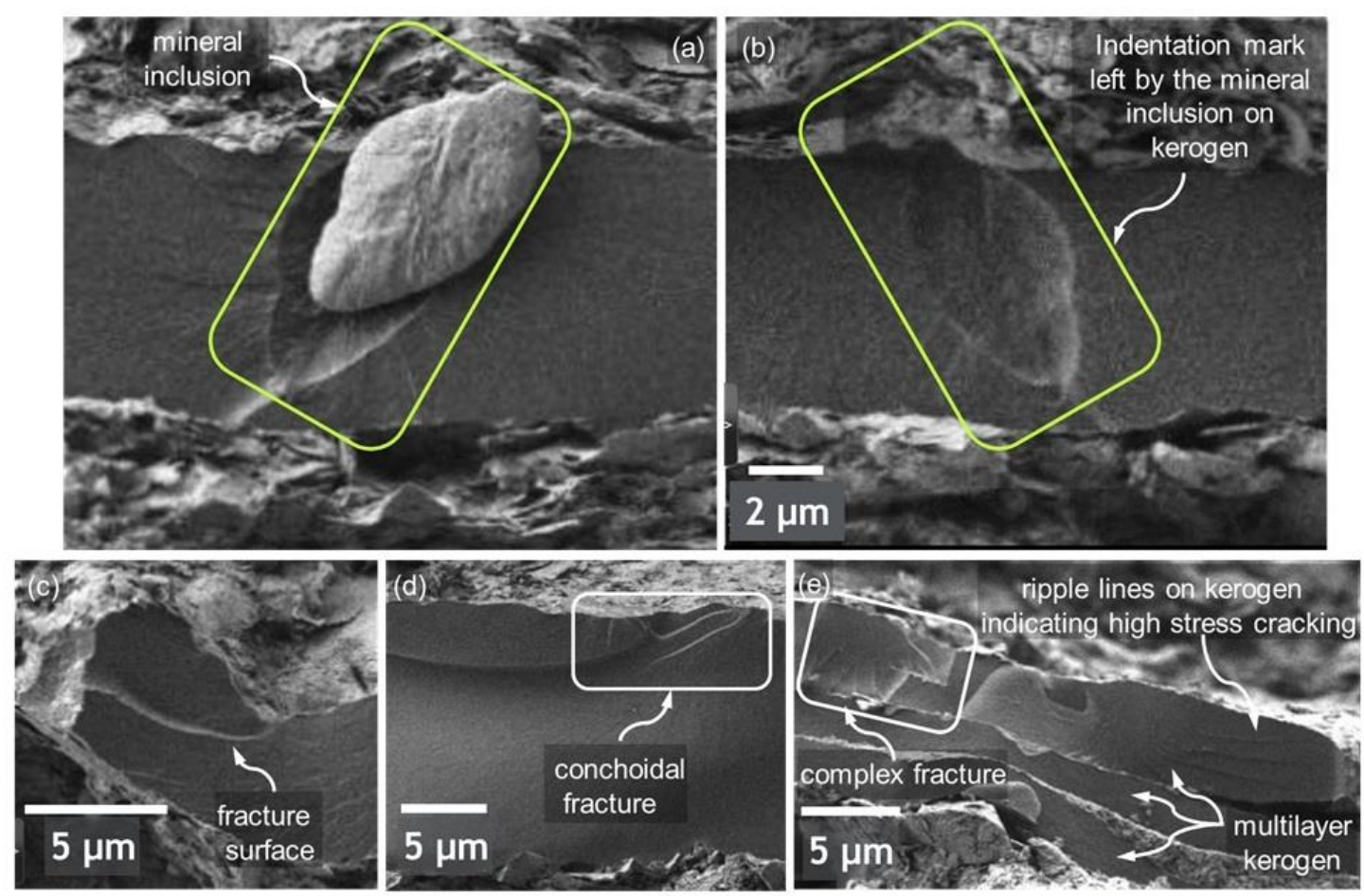

Figure 2. Glassy fracture surface of a single layer kerogen resulting from a pre-existing inclusion $(a, b)$. Fractography of multi-layer kerogen bodies showing fracture surfaces $(c, d, e)$, and obsidian fracture (d, e).

\section{References}

[1] Abousleiman, Y. et al., Acta Geotechnica. 11, 573-594, 2016.

[2] Haque, M.H. et al., American Geophysical Union, Fall Meeting Abstract \#MR44A-08. Dec 2017.

[3] Vandenbroucke, M. et al., Organic Geochemistry. 38, 719-833, 2007.

[4] Haque, M.H. et al., EMI Conference. MS 104, Multiscale Mechanics and Physics of Shale. Jun 2017. 